\title{
Quantum-behaved Particle Swarm Optimization for Multiple-fuel-constrained Generation Scheduling of Power System
}

\author{
Chao-Lung Chiang \\ Depart. of Electronic Engineering, Nan Kai University of Technology, Nan-Tou County, Taiwan.
}

\begin{abstract}
This research proposes a quantum-behaved particle swarm optimization with a multiplier updating technique (QPSOMU) for the multiple-fuel-constrained generation scheduling of power system. The quantum-behaved particle swarm optimization (QPSO) equips with a migration can efficiently search and actively explore solutions. The multiplier updating (MU) is introduced to avoid deforming the augmented Lagrange function and resulting in difficulty of solution searching. The proposed algorithm integrates the QPSO and the MU that has merits of automatically adjusting the randomly given penalty to a proper value and requiring only a small-size population for the power economic dispatch problem of the multiple-fuel-constrained generation scheduling. Numerical results of two test systems indicate that the proposed algorithm is more suitable than previous approaches in the practical economic dispatch for the multiple-fuel-constrained generation scheduling of power system.
\end{abstract}

Keywords-quantum-behaved particle swarm optimization; generation scheduling; multiple-fuel-constrained; power system

\section{INTRODUCTION}

The power economic dispatch (PED) problem involves allocation of generations to different thermal units to minimize the cost of generation, while satisfying the equality and inequality constraints of the power system [1]. In general, the economic dispatch problem aims to increase utilization at the lowest cost of fuel [2], [3]. Generally, the fuel cost function of a generator has been approximately represented by a single quadratic function. However, many generating units, particularly those which are supplied with multi-fuel sources (coal, nature gas, or oil), lead to the problem of determining the most economic fuel to burn. Some studies of the PED problems, such as an efficient crisscross optimization (CSO) [4], double weighted particle swarm optimization (DWPSO) [5], lightning flash algorithm (LFA) [6], numerical method (NM) [7], shuffled frog leaping algorithm and global-best harmony search (SFLAGHS) [8], adaptive Hopfield neural network (AHNN) [9], evolutionary programming technique (EP) [10], augmented Lagrange Hopfield network initialized quadratic programming (QP-ALHN) [11], and an improved particle swarm optimization with a dynamic search space squeezing strategy [12] have considered the multiple-fuel-constrained generation scheduling of power systems.

Particle swarm optimization (PSO) [13] [16] has been widely used in dealing with many real-world problems because of its simplicity and facile realization. However, owing to the restricted velocity, the searching area of a particle is limited and diminishing in PSO. Which means, in a PSO system, the searching space cannot cover the whole feasible region and global convergence cannot be guaranteed [17]. This is also the main cause of the premature in PSO. In order to dispose of the disadvantages of PSO, quantum-behaved particle swarm optimization (QPSO) [17] [23] is proposed for solving the PED problems.

\section{PROBLEM FORMULATION}

The PED problem can be described as an optimization (minimization) process with objective [1]:

$$
\text { Minimize } \quad \sum_{i=1}^{n_{p}} F_{i}\left(P_{i}\right)
$$

Where $F_{i}\left(P_{i}\right)$ is the fuel cost function of the $i^{\text {th }}$ unit, $P_{i}$ is the power generated by the $i^{\text {th }}$ unit, and $n_{P}$ is the number of dispatchable units. Subject to the equality constraint of the power balance as:

$$
\sum_{i=1}^{n_{p}} P_{i}=P_{d}+P_{L}
$$

Where $P_{d}$ is the system load demand and $P_{L}$ is the transmission loss, and generating capacity constraints as:

$$
P_{i}^{\min } \leq P_{i} \leq P_{i}^{\max }, \quad i=1, \cdots, n_{p}
$$

Where $P_{i}^{\min }$ and $P_{i}^{\max }$ are the minimum and maximum power outputs of the $i^{\text {th }}$ unit. The cost function addressing valve-point loadings of generating units is accurately represented as [24]:

$$
F_{i}\left(P_{i}\right)=a_{i}+b_{i} P_{i}+c_{i} P_{i}^{2}+\left|e_{i} \times \sin \left(f_{i} \times\left(P_{i}^{\min }-P_{i}\right)\right)\right|
$$

Where $a_{i}, b_{i}$, and $c_{i}$ are the fuel cost coefficients of the $i^{\text {th }}$ unit, and $e_{i}$ and $f_{i}$ are fuel cost coefficients of the $i^{t h}$ unit with valve- 
points effects. For considering the multiple fuel option, the fuel cost function should be practically expressed as [7]:

$$
F_{i}\left(P_{i}\right)=\left\{\begin{array}{llll}
a_{i 1}+b_{i 1} P_{i}+c_{i 1} P_{i}^{2}, & \text { fuel } & 1, & P_{i}^{\min } \leq P_{i} \leq P_{i 1} \\
a_{i 2}+b_{i 2} P_{i}+c_{i 2} P_{i}^{2}, & \text { fuel } & 2, & P_{i 1}<P_{i} \leq P_{i 2} \\
\vdots & & \vdots \\
a_{i k}+b_{i k} P_{i}+c_{i k} P_{i}^{2}, & \text { fuel } & k, & P_{i k-1}<P_{i} \leq P_{i}^{\max }
\end{array}\right.
$$

Where $a_{i k}, b_{i k}$, and $c_{i k}$ are cost coefficients of the $i^{\text {th }}$ generator using the fuel type $k$. To obtain an accurate and practical economic dispatch solution, the realistic operation of the PED problem should be considered both valve-point effects and multiple fuels. This paper proposed an incorporated cost model, which combines the valve-point loadings and the fuel changes into one frame. Therefore, the cost function should combine (4) with (5), and can be realistically represented as [3]:

$$
F_{i}\left(P_{i}\right)=\left\{\begin{array}{c}
a_{i 1}+b_{i 1} P_{i}+c_{i 1} P_{i}^{2}+\left|e_{i 1} \times \sin \left(f_{i 1} \times\left(P_{i 1}^{\min }-P_{i 1}\right)\right)\right| \\
\text { for fuel } 1, \quad P_{i}^{\min } \leq P_{i} \leq P_{i 1} \\
a_{i 2}+b_{i 2} P_{i}+c_{i 2} P_{i}^{2}+\left|e_{i 2} \times \sin \left(f_{i 2} \times\left(P_{i 2}^{\min }-P_{i 2}\right)\right)\right| \\
\text { for fuel } 2, \quad P_{i 1}<P_{i} \leq P_{i 2}(6) \\
\vdots \\
a_{i k}+b_{i k} P_{i}+c_{i k} P_{i}^{2}+\left|e_{i k} \times \sin \left(f_{i k} \times\left(P_{i k}^{\min }-P_{i k}\right)\right)\right| \\
\text { for fuel } k, \quad P_{i k-1}<P_{i} \leq P_{i}^{\max }
\end{array}\right.
$$

Complication of the actual PED problem is due to the incorporated cost model composed of both valve-point effects and multiple fuels. Hence, an algorithm that overcomes these complexities has to be evolved.

\section{THE PROPOSED ALGORITHM}

\section{A. The QPSO}

In QPSO, the position of a particle is depicted by its local attractor and a probability density function. In this case, the particles in QPSO have got rid of the limitation of trajectory. Analysis in [17] shows that QPSO is global convergent. Another advantage is that there is only one parameter in QPSO. Hence, QPSO is very easy to implement. More details of the QPSO used in the field of power system have shown in [18] [23].

\section{B. The $M U$}

Considering the nonlinear problem with general constraints as follows: Where $h_{k}(x)$ and $g_{k}(x)$ stand for equality and inequality constraints, respectively.

$$
\begin{array}{lll} 
& \min _{x} f(x) & \\
\text { subject to } \quad & h_{k}(x)=0, \quad k=1, \ldots, m_{e} \\
& g_{k}(x) \leq 0, \quad k=1, \ldots, m_{i}
\end{array}
$$

Where $x$ represents a $n_{C}$-dimensional variable, and the $h_{k}(x)$ and $g_{k}(x)$ stand for equality and inequality constraints, respectively. The penalty function method is frequently applied to manage constraints in evolutionary algorithms. Such a technique converts the primal constrained problem into an unconstrained problem by penalizing constraint violations. The penalty function method is simple in concept and implementation. However, its primal limitation is the degree to which each constraint is penalized. These penalty terms have certain weaknesses that become fatal when penalty parameters are large. Such a penalty function tends to be ill conditioned near the boundary of the feasible domain where the optimum point is usually located.

Lagrange method can markedly overcome the drawbacks of the penalty method. The augmented Lagrange function (ALF) [25] for constrained optimization problems is defined as:

$$
\begin{aligned}
& L_{a}(x, v, v)=f(x)+ \\
& \sum_{k=1}^{m_{e}} \alpha_{k}\left\{\left[h_{k}(x)+v_{k}\right]^{2}-v_{k}^{2}\right\}+\sum_{k=1}^{m_{i}} \beta_{k}\left\{\left\langle g_{k}(x)+v_{k}\right\rangle_{+}^{2}-v_{k}^{2}\right\}
\end{aligned}
$$

Where $\alpha_{k}$ and $\beta_{k}$ are the positive penalty parameters, and the corresponding Lagrange multipliers $v=\left(v_{1}, \ldots, v_{m_{e}}\right)$ and $v=\left(v_{1}, \ldots, v_{m_{i}}\right) \geq 0$ are associated with equality and inequality constraints, respectively.

The contour of the augmented Lagrange function does not change shape from generation to generation while constraints are linear. Therefore, the contour of the augmented Lagrange function is simply shifted or biased in relation to that of the original objective function, $f(x)$. Consequently, small penalty parameters can be used in the MU. However, the shape of contour of $L_{a}$ is changed by penalty parameters while the constraints are nonlinear, demonstrating that the computational difficulties of using large penalty parameters for nonlinear constraints remain. The adaptive penalty parameters are employed to alleviate the above difficulties. More details of the MU have shown in [26].

\section{The Proposed QPSO-MU}

The ALF is used to obtain a minimum value in the inner loop with the given penalty parameters and multipliers, which are then updated in the outer loop toward producing an upper limit of $L_{a}$. When both inner and outer iterations become sufficiently large, the ALF converges to a saddle-point of the dual problem [27].

\section{SYSTEM SimUlATIONS}

This section employs two examples to illustrate the effectiveness of the proposed QPSO-MU with respect to the quality of the solution obtained. The first example considers with multi-fuel effects and without the valve-point loadings. The other example considers both valve-point effects and multiple fuels. The MU algorithm was used in QPSO to hand the equality and inequality constraints for the two tests. The computation was implemented on a personal computer (Intel(R) Core(TM) i73770 CPU @ 3.4 GHz with 8G Ram) in FORTRAN-90 language. Setting factors were used identically in the two tests as follows; 
the population size is set as 5 . The iteration numbers of outer loop and inner loop are set to (outer, inner) as (10, 3000). The proposed approach solves PED problems considering system constraints of power balance (2) and capacity limits (3).

\section{A. Example 1}

The proposed algorithm was employed to solve the multiplefuel-constrained generation scheduling of power system with the cost model (5). This example system contained ten dispatching units addressing multiple fuels for a load demand of $2500 \mathrm{MW}$. The system data of this example are all the same as [7]. The implementation of this examples can be described as follows:

$$
L_{a}(x, v, v)=f(x)+\alpha_{1}\left\{\left[h_{1}(x)+v_{1}\right]^{2}-v_{1}^{2}\right\}
$$

$$
\text { objective : } \min _{x=\left(P_{1}, P_{2}, \ldots, P_{10}\right)} f(x)=\sum_{i=1}^{10} F_{i}\left(P_{i}\right)
$$

$$
\text { subject to } h_{1}: \quad \sum_{i=1}^{10} P_{i}-P_{d}=0
$$

This example compares the proposed QPSO-MU with five previous approaches of NM [7], SFLA-GHS [8], AHNN [9], EP [10], and QP-ALHN [11]. Compared results are listed in Table I, where $T P, T C$, and $F T$ stand the total power, total cost, and the fuel types respectively. Not only do the results obtained by the proposed QPSO-MU supply sufficient load demand, but the results obtained by the proposed method have lower costs than that obtained by the previous approaches. This example reveals that the QPSO-MU is more suitable than previous methods in application because the proposed algorithm is easy to implement, and has a satisfactory result of solving the PED problem with multiple-fuel-constrained generation scheduling.

TABLE I. COMPUTATIONAL RESULTS OF THE PROPOSED QPSO-MU AND FIVE PREVIOUS METHODS FOR EXAMPLE 1.

\begin{tabular}{|c|c|c|c|c|c|c|c|c|c|c|c|c|}
\hline \multirow{2}{*}{ Unit } & \multicolumn{2}{|c|}{ NM [7] } & \multicolumn{2}{c|}{ SFLA-GHS [8] } & \multicolumn{2}{c|}{ AHNN [9] } & \multicolumn{2}{c|}{ EP [10] } & \multicolumn{2}{c|}{ QP-EALHN [11] } & \multicolumn{2}{c|}{ QPSO-MU } \\
\cline { 2 - 15 } & $F T$ & Gen & $F T$ & Gen & $F T$ & Gen & $F T$ & Gen & $F T$ & Gen & $F T$ & Gen \\
\hline 1 & 2 & 206.6 & 2 & 206.5190 & 2 & 206.7 & 2 & 206.7 & 2 & 206.518 & 2 & 206.5188 \\
\hline 2 & 1 & 206.5 & 1 & 206.4573 & 1 & 205.8 & 1 & 205.8 & 1 & 206.457 & 1 & 206.4573 \\
\hline 3 & 1 & 265.9 & 1 & 265.7391 & 1 & 265.6 & 1 & 265.6 & 1 & 265.739 & 1 & 265.7392 \\
\hline 4 & 3 & 236.0 & 3 & 235.9531 & 3 & 235.8 & 3 & 235.8 & 3 & 235.953 & 3 & 235.9531 \\
\hline 5 & 1 & 258.2 & 1 & 258.0177 & 1 & 258.2 & 1 & 258.2 & 1 & 258.018 & 1 & 258.0178 \\
\hline 6 & 3 & 236.0 & 3 & 235.9531 & 3 & 235.8 & 3 & 235.8 & 3 & 235.953 & 3 & 235.9531 \\
\hline 7 & 1 & 269.0 & 1 & 268.8635 & 1 & 269.4 & 1 & 269.4 & 1 & 268.863 & 1 & 268.8636 \\
\hline 8 & 3 & 236.0 & 3 & 235.9531 & 3 & 235.8 & 3 & 235.8 & 3 & 235.953 & 3 & 235.9531 \\
\hline 9 & 1 & 331.6 & 1 & 331.4877 & 1 & 330.1 & 1 & 330.1 & 1 & 331.487 & 1 & 331.4876 \\
\hline 10 & 1 & 255.2 & 1 & 255.0562 & 1 & 256.9 & 1 & 256.9 & 1 & 255.058 & 1 & 255.0564 \\
\hline$T P(\mathrm{MW})$ & 2501.0 & 2499.9998 & \multicolumn{2}{|c|}{2500.1} & \multicolumn{2}{|c|}{2500.1} & & 2499.999 & & 2500.0000 \\
\hline \multicolumn{2}{|c|}{$T C(\$)$} & 526.70 & 526.2388 & \multicolumn{2}{|c|}{526.23} & \multicolumn{2}{|c|}{526.23} & & 526.239 & 526.238760 \\
\hline
\end{tabular}

\section{B. Example 2}

This test system considers both multiple fuel options and valve-point effects with the cost model (6) for a load demand of $2700 \mathrm{MW}$. The system data of this example are given in [5]. The implementation of the proposed algorithm of this example are identical to the example 1, as (9) (11). The comparisons between the proposed algorithm with MSFLA [8], CCEDE [28], RCGA [29], SPPO [3], CSO [4], and DWPSO [5] are illustrated in Table II, and the proposed QPSO-MU exhibits not only better solution quality but also acquire an exact $T P$ for load demand than the previous papers. Although DWPSO [5] has a less TC (\$622.7333) but the TP (2697.0000 MW) is short of 3MW for

\begin{tabular}{|c|c|c|c|c|c|c|c|c|c|c|c|c|c|c|}
\hline \multirow{2}{*}{ Unit } & \multicolumn{2}{|c|}{ MSFLA [8] } & \multicolumn{2}{|c|}{ CCEDE [28] } & \multicolumn{2}{|c|}{ RCGA [29] } & \multicolumn{2}{|c|}{ SPPO [3] } & \multicolumn{2}{|c|}{ CSO [4] } & \multicolumn{2}{|c|}{ DWPSO [5] } & \multicolumn{2}{|c|}{ QPSO-MU } \\
\hline & $F T$ & Gen & $F T$ & Gen & $F T$ & Gen & $F T$ & Gen & $F T$ & Gen & $F T$ & Gen & $F T$ & Gen \\
\hline 1 & 2 & 215.49912 & 2 & 218.5940 & 2 & 218.5939 & 2 & 218.10140 & 2 & 218.1049 & 2 & 212.5155 & 2 & 218.2328 \\
\hline 2 & 1 & 210.72163 & 1 & 210.9690 & 1 & 211.2166 & 1 & 209.90700 & 1 & 211.9071 & 1 & 209.9788 & 1 & 211.8571 \\
\hline 3 & 1 & 284.71457 & 1 & 280.6571 & 1 & 280.6571 & 1 & 280.65870 & 1 & 280.6570 & 1 & 272.5913 & 1 & 280.7009 \\
\hline 4 & 3 & 239.77379 & 3 & 239.6394 & 3 & 239.3707 & 3 & 239.82070 & 3 & 239.4176 & 3 & 241.7894 & 3 & 239.6262 \\
\hline 5 & 1 & 286.44651 & 1 & 279.9345 & 1 & 279.9345 & 1 & 279.94060 & 1 & 279.9345 & 1 & 272.8177 & 1 & 278.4674 \\
\hline 6 & 3 & 240.17688 & 3 & 239.7738 & 3 & 239.3707 & 3 & 239.52670 & 3 & 239.6609 & 3 & 240.3113 & 3 & 239.6262 \\
\hline 7 & 1 & 278.86514 & 1 & 287.7275 & 1 & 287.7275 & 1 & 287.69920 & 1 & 287.7274 & 1 & 292.4608 & 1 & 288.5558 \\
\hline 8 & 3 & 242.05715 & 3 & 239.7738 & 3 & 239.5051 & 3 & 240.08930 & 3 & 239.6863 & 3 & 241.5206 & 3 & 239.6262 \\
\hline 9 & 3 & 425.31813 & 3 & 427.0623 & 3 & 427.7553 & 3 & 426.39040 & 3 & 427.0352 & 3 & 433.9807 & 3 & 428.4696 \\
\hline 10 & 1 & 276.42708 & 1 & 275.8686 & 1 & 275.8686 & 1 & 275.86600 & 1 & 275.8686 & 1 & 279.0339 & 1 & 274.8378 \\
\hline \multicolumn{2}{|c|}{$T P(\mathrm{MW})$} & 2700.00000 & \multicolumn{2}{|c|}{2700.0000} & \multicolumn{2}{|c|}{2700.0000} & \multicolumn{2}{|c|}{2698.00000} & \multicolumn{2}{|c|}{2699.9995} & \multicolumn{2}{|c|}{2697.0000} & \multicolumn{2}{|c|}{2700.0000} \\
\hline \multicolumn{2}{|c|}{$T C(\$)$} & 624.11569 & \multicolumn{2}{|c|}{623.8288} & \multicolumn{2}{|c|}{623.8281} & \multicolumn{2}{|r|}{82790} & \multicolumn{2}{|c|}{623.8237} & \multicolumn{2}{|c|}{622.7333} & \multicolumn{2}{|c|}{623.80932} \\
\hline
\end{tabular}
power balance.

TABLE II. COMPUTATIONAL RESULTS OF THE PROPOSED QPSO-MU AND SIX PREVIOUS METHODS FOR EXAMPLE 2. 


\section{CONCLUSIONS}

The realistic PED problem is complicated because change fuels must be considered. The QPSO helps the proposed algorithm efficiently search and explore. The MU helps the proposed method avoid deforming the augmented Lagrange function and resulting in difficulty of solution searching. The proposed method integrates the QPSO and MU such that it has merits of automatically adjusting the randomly given penalty to a proper value and requiring only a small-size population. Numerical results of the two examples demonstrate that the proposed method has more advantages for solving the PED problems with multiple-fuel-constrained generation scheduling than previous approaches for power operations.

\section{REFERENCES}

[1] Y. Yu and P. Luh, "Scalable corrective security-constrained economic dispatch considering conflicting contingencies," International Journal of Electrical Power \& Energy Systems, vol. 98, 2018, pp. 269-278.

[2] Q. Qin, S. Cheng, X. Chu, X. Lei, and Y. Shi, "Solving non-convex/nonsmooth economic load dispatch problems via an enhanced particle swarm optimization," Applied Soft Computing, vol. 59, 2017, pp. 229-242.

[3] N. J. Singh, J. S. Dhillon, and D. P. Kothari, "Synergic predator-prey optimization for economic thermal power dispatch problem," Applied Soft Computing, vol. 43, 2016, pp. 298-311.

[4] A. Meng, J. Li, H. Yin, "An efficient crisscross optimization solution to largescale non-convex economic load dispatch with multiple fuel types and valve-point effects," Energy, vol. 113, 2016, pp. 1147-1161.

[5] M. Kheshti, L. Ding, S. Ma, and B. Zhao, "Double weighted particle swarm optimization to non-convex wind penetrated emission/economic dispatch and multiple fuel option systems," Renewable Energy, vol. 125, 2018, pp. 1021-1037.

[6] M. Kheshti, X. Kang, Z. Bie, Z. Jiao, and X Wang, "An effective Lightning Flash Algorithm solution to large scale non-convex economic dispatch with valve-point and multiple fuel options on generation units," Energy, vol. 129, 2017, pp. 1-15.

[7] C. E. Lin and G. L. Viviani, "Hierarchical economic dispatch for piecewise quadratic cost functions, "IEEE Transactions on PAS. Vol. 103, no. 6, 1984, pp. 1170-1175.

[8] K. Vaisakha and A. Srinivasa Reddy, "MSFLA/ GHS/ SFLA-GHS/ SDE algorithms for economic dispatch problem considering multiple fuels and valve point loadings," Applied Soft Computing, vol. 13, 2013, pp. 42814291.

[9] K. Y. Lee, A. S. Yome, and J. H. Park, "Adaptive Hopfield neural networks for economic load dispatch," IEEE Transactions on Power Systems, vol. 13, no. 2, 1998, pp. 519-526.

[10] T. Jayabarathi and G. Sadasivam, "Evolutionary programming- based economic dispatch for units with multiple fuel options, "European Trans. on Electrical Power, vol.10, no. 3, 2000, pp. 167-170.

[11] D. N. Vo and P. Schegner, "Augmented lagrange hopfield network initialized by quadratic programming for economic dispatch with piecewise quadratic cost functions and prohited zones, "Applied Soft Computing, vol. 13, 2013, pp. 292-301.

[12] A. K. Barisal, "Dynamic search space squeezing strategy based intelligent algorithm solutions to economic dispatch with multiple fuels," International Journal of Electrical Power and Energy Systems, vol. 45, 2013, pp. 50-59.

[13] J. Kennedy and R. Eberhart, "A new optimizer using particle swarm theory," Proceedings of the Sixth International Symposium on Micro Machine and Human Science, 1995, pp. 39-43.

[14] N. K. Rout, D. P. Das, and G. Panda, "PSO based adaptive narrowband ANC algorithm without the use of synchronization signal and secondary path estimate," Mechanical Systems and Signal Processing, vol. 114, 2019, pp. 378-398.
[15] H. Yu, Y. Tan, J. Zeng, C. Sun, and Y. Jin, "Surrogate-assisted hierarchical particle swarm optimization," Information Sciences, vol. 454455, 2018, pp. 59-72.

[16] R. George and P. Samuel, "Fixing class design inconsistencies using self regulating particle swarm optimization," Information and Software Technology, vol. 99, 2018, pp. 81-92.

[17] J. Sun, X. Xu, V. Palade, W. Fang, C. Lai and W. Xu, "Convergence analysis and improvements of quantum-behaved particle swarm optimization," Information Sciences, vol. 193, no. 15, 2012, pp. 81-103.

[18] T. Liu, L. C. Jiao, W. P. Ma and R. H. Shang, "Quantum-behaved particle swarm optimization with collaborative attractors for nonlinear numerical problems," Commun. Nonlinear Sci. Numer. Simulat., vol. 44, 2017, pp. 167-183.

[19] Y. Y. Li, X. Bai, L. C. Jiao and Y. Xue, "Partitioned-cooperative quantumbehaved particle swarm optimization based on multilevel thresholding applied to medical image segmentation," Applied Soft Computing, vol. 56, 2017, pp. 345-356.

[20] H. Luo, P. Jia, S. Qiao, and S. Duan, "Enhancing electronic nose performance based on a novel QPSO-RBM technique," Sensors and Actuators B: Chemical, vol. 259, 2018, pp. 241-249.

[21] W. Gan, D. Zhu, and D. Ji, "QPSO-model predictive control-based approach to dynamic trajectory tracking control for unmanned underwater vehicles," Ocean Engineering, vol. 158, 2018, pp. 208-220.

[22] C. P. Mohanty, S. S. Mahapatra, and M. R. Singh, "An intelligent approach to optimize the EDM process parameters using utility concept and QPSO algorithm," Engineering Science and Technology, an International Journal, vol. 20, Issue 2, 2017, pp. 552-562.

[23] K. Meng, H. G. Wang, Z. Y. Dong, and K. P.Wo, "Quantum-Inspired Particle Swarm Optimization for Valve-Point Economic Load Dispatch”, IEEE Trans. Power Syst., vol. 25, issue. 1, 2010, pp. 215-222.

[24] S. Ninha, R. Chakrabarti, and P. K. Chattopadhyay, "Evolutionary programming techniques for economic load dispatch", IEEE Evol. Comput., vol. 7, no. 1, 2003, pp. 83-94.

[25] M. J. D. Powell, "Algorithms for nonlinear constraints that use Lagrangian function," Math. Programming, vol. 14, pp. 224-248, 1978.

[26] C. L. Chiang, C. T. Su, and F. S. Wang, "Augmented Lagrangian method for evolutionary optimization of mixed-integer nonlinear constrained problems," Intern. Math. J., vol.2, no. 2, pp. 119-154, 2002.

[27] Z. Michalewicz and M. Schoenauer, "Evolutionary algorithms for constrained parameter optimization problems," Evolutionary Computation, vol. 4, no. 1, pp. 1-32, 1996.

[28] M. Ghasemi, M. Taghizadeh, S. Ghavidel, and A. Abbasian, "Colonial competitive differential evolution: An experimental study for optimal economic load dispatch," Applied Soft Computing, vol. 40, 2016, pp. 342-363.

[29] N. Amjady, H. Nasiri-Rad, "Nonconvex economic dispatch with AC constraints by a new real coded genetic algorithm," IEEE Transactions on Power Systems, vol. 24, 2009, pp. 1489-1502. 